\title{
Process and Outcomes of Patient-Centered Medical Care With Alaska Native People at Southcentral Foundation
}

\author{
David L. Driscoll, PbD, MPH, $M A^{1}$ \\ Vanessa Hiratsuka, MPH ${ }^{2}$ \\ Janet M. Jobuston, $P b D^{1}$ \\ Sara Norman ${ }^{2}$ \\ Katie M. Reilly, $M P H^{1}$ \\ Jennifer Shaw, MA \\ Julia Smith, MS \\ Quenna N. Szafran, BS, RN ${ }^{1}$ \\ Denise Dillard, $\mathrm{PbD}^{2}$ \\ 'University of Alaska, Anchorage, Alaska \\ ${ }^{2}$ Southcentral Foundation, Anchorage, \\ Alaska

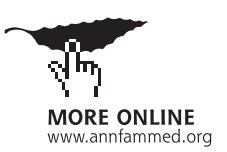

Conflicts of interest: authors report none.

\section{CORRESPONDING AUTHOR}

David L. Driscoll, PhD, MPH, MA Institute for Circumpolar Health Studies University of Alaska Anchorage 3211 Providence Dr, DPL 404

Anchorage, AK 99508

DavidDriscoll@uaa.alaska.edu

\begin{abstract}
PURPOSE This study describes key elements of the transition to a patient-centered medical home (PCMH) model at Southcentral Foundation (SCF), a tribally owned and managed primary care system, and evaluates changes in emergency care use for any reason, for asthma, and for unintentional injuries, during and after the transition.
\end{abstract}

METHODS We conducted a time series analyses of emergency care use from medical record data. We also conducted 45 individual, in-depth interviews with PCMH patients (customer-owners), primary care clinicians, health system employees, and tribal leaders.

RESULTS Emergency care use for all causes was increasing before the PCMH implementation, dropped during and immediately after the implementation, and subsequently leveled off. Emergency care use for adult asthma dropped before, during, and immediately after implementation, subsequently leveling off approximately 5 years after implementation. Emergency care use for unintentional injuries, a comparison variable, showed an increasing trend before and during implementation and decreasing trends after implementation. Interview participants observed improved access to primary care services after the transition to the PCMH tempered by increased staff fatigue. Additional themes of PCMH transformation included the building of relationships for coordinated, team-based care, and the important role of leadership in PCMH implementation.

CONCLUSIONS All reported measures of emergency care use show a decreasing trend after the PCMH implementation. Before the implementation, overall use and use for unintentional injuries had been increasing. The combined quantitative and qualitative results are consistent with decreased emergency care use resulting from a decreased need for emergency care services due to increased availability of primary care services and same-day appointments.

Ann Fam Med 2013;11:S41-S49. doi:10.1370/afm.1474.

\section{INTRODUCTION}

laska Native and American Indian people experience poorer health
outcomes than the overall US population.' Access to quality health
care represents one component of a comprehensive program to reduce health disparities in these populations. The patient-centered medical home (PCMH) model has been shown to improve primary care quality, access, and health outcomes ${ }^{2}$ and is postulated to be an effective approach for reducing health disparities in preventive and chronic disease care. ${ }^{3}$

Southcentral Foundation (SCF) is an Alaska Native-owned, nonprofit organization serving nearly 60,000 Alaska Native and American Indian people living in Southcentral Alaska with the mission "to work with the Native Community to achieve wellness through health and related services." In 1997, Alaska Native and American Indian residents of south- 


\section{Figure 1. Timeline for transition to Nuka System of Care.}

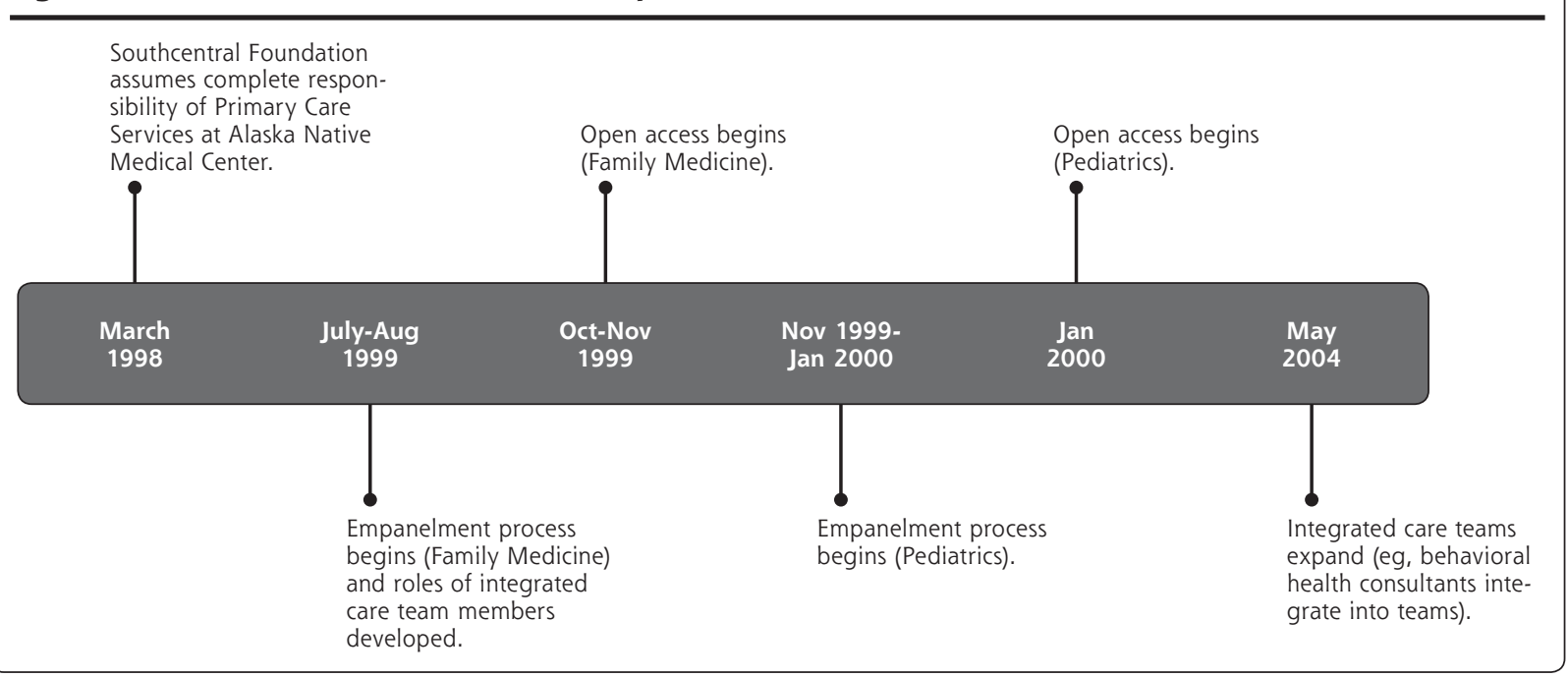

central Alaska began receiving care at new hospital and outpatient facilities at the Alaska Native Medical Center (ANMC). In 1998, SCF assumed responsibility for primary care services at ANMC after more than 50 years of management by the Indian Health Service (IHS). ${ }^{4}$ SCF provides care for Alaska Native and American Indian people in Anchorage, the MatanuskaSusitna Borough, and 50 rural villages in the region.

In 1999, SCF leadership began implementing key components of the PCMH model, as shown in Figure 1. The tailored model, called the Nuka System of Care, is based on several key characteristics of a $\mathrm{PCMH}$, including patient match to an integrated and comprehensive care team (called empanelment by SCF), enhanced access to health care (open access), and care coordination by integrated primary care teams (team-based care). ${ }^{5}$

- Empanelment: Patients are matched, either by self-selection or assignment, to an integrated and comprehensive care team (ICT). Patients schedule primary care appointments with their team members.

- Open access: To the extent possible, patients' barriers to access are mitigated through open scheduling, expanded office hours, and increased availability of electronic communication between patients and ICT members. ${ }^{4}$

- Team-based care: Coordinated care is delivered by multidisciplinary teams rather than by individual clinicians. ${ }^{4}$ These teams include primary care physicians or physician assistants, nurses, certified medical assistants, and other clinicians. Over time, behavioral health consultants, nutritionists, and appointment schedulers were added.

Although detailed information about changes in the number of clinicians and other ICT members with implementation of the PCMH model are not available, SCF reported that health care service expenses increased by a factor of 3.8 from 1998 to 2009. In addition to increasing the workforce during this time, SCF also made major investments in the construction of new clinical facilities, and in employee training and development. In 2010, SCF received the National Committee for Quality Assurance's primary care center PCMH accreditation. Further information relevant to understanding and transporting findings from this study can be found in the Supplemental Appendix (available online at http://annfammed.org/content/11/ Suppl_1/S41/suppl/DC1).

Although the term medical home was first used by the American Academy of Pediatrics in 1967, medical home principles were not widely adopted outside the field of pediatrics until 2002. ${ }^{6}$ The SCF PCMH transition began in 1999 following recommendations provided by patients, employees, and tribal leadership. This early adoption of the SCF PCMH into the primary care setting provided the research team with more than a decade of secondary data on health care process and outcomes to analyze. As increasing demand steadily outpaces resources in the current health care climate, such a study is needed in Alaska Native and American Indian communities and more broadly to determine if and how PCMH models of care effectively impact health care quality, experiences, costs, and outcomes.

\section{METHODS}

\section{Mixed Methods Approach}

This article describes a mixed method examination of the SCF PCMH model of primary care. We collected 
aggregate medical record data to describe and discuss changes in health care system outcomes, including service use, within emergency care overall, as well as for asthma and for unintentional injuries such as falls and car accidents. We looked in more detail at the effects on adult asthma care because adult asthma has been identified by the Agency for Healthcare Research and Quality (AHRQ) as an ambulatory care-sensitive condition, or one for which outpatient care can reduce the need for emergency care and hospitalization. We examined changes in emergency care use for unintentional injury, for which outpatient care is less likely to have an effect, as a comparison measure. We also collected semistructured, individual interview responses to examine the implementation of the PCMH with relevance to the quantitative data from the perspectives of patients, health care professionals, other employees, and tribal leaders. These data were combined to assess prospective associations between the trends identified in the quantitative findings and the processes and experiences described in the qualitative findings.

Before starting data collection, we developed a mixed methods analytic plan. This plan, referred to as the analytic cross-walk, linked secondary data measures with responses to specific semistructured interview questions. For example, it linked the rate of emergency care visits per year with clinician and other employee responses to the question "How have hospitalizations changed since empanelment?" and patient responses to the question "How have your emergency care visits changed since the transformation?" The cross-walk further linked secondary data measures as to the treatment rates for a variety of primary caresensitive health conditions with clinician responses to the questions "Can you tell me how diagnosis rates may have changed" or "Which conditions/health issues were most or least affected by [the transition to a $\mathrm{PCMH}$ ?" and patient responses to the question "What were some of the strengths/weaknesses of the health care system [during both time periods]?"

We combined the quantitative and qualitative data in a straightforward sequential mixed methods design ${ }^{7}$ in which the secondary, quantitative data were collected and analyzed before the primary, qualitative data. This approach allowed us to focus the qualitative analytic process on key changes in outcomes or use of various components of the SCF health care system throughout the study period. It also allowed us to assess how changes in health care system outcomes and service use could be explained by, or otherwise associated with, the process by which these changes have taken place in the system from the perspective of patients, their clinicians and other employees, and the tribal leadership at SCF.
All study protocols were approved by the IHS Alaska Area and University of Alaska Anchorage institutional review boards, and tribal leadership of SCF and the Alaska Native Tribal Health Consortium.

\section{Quantitative Data Collection and Analysis}

The goal for the quantitative data analysis was to describe and discuss changes in health care system outcomes including service use before, during, and after PCMH implementation at SCF. To do that, we examined secondary data to assess changes over time in monthly rates of clinic visits, emergency care, and hospitalization overall and for chronic conditions considered sensitive to primary care. ${ }^{8}$ We also examined changes in emergency care and hospitalization for unintentional injuries as a comparison measure. Because of space limitations, we are reporting on only 3 emergency care measures here $e_{i}$ other results from the secondary analysis will be reported later.

To evaluate changes in health care use and outcomes related to the PCMH implementation that started in 1999, we analyzed monthly or bimonthly rates starting in March 1996. To calculate rates, we extracted monthly event counts and patient counts from the Resource and Patient Management System (RPMS), SCF's electronic health record system. Data were provided to the analytic team at the group level without individual-level details.

The ANMC provides services to all IHS beneficiaries including Alaska Native and American Indian people who live in the Anchorage area and receive primary care services from SCF and those who live in other areas of the state who may be transported to ANMC for tertiary care.

The sampling frame consisted of all individuals who sought care at ANMC, excluding visits coded as pharmacy, telephone, or administrative, during the 36 months before the month for which data were pulled. To limit the analysis to patients who received primary care from SCF, we limited the sampling population to individuals with a city of residence in the Anchorage area. RPMS retains the most recent city of residence; therefore, residence was based on the city of residence at the time of data extraction.

SCF refers to their Alaska Native and American Indian patients as customer-owners (COs) because they are both consumers of health care and, as tribal members, also owners of the health care system. There are a small number of 2 other groups represented in these data: Commissioned Corps officers, eligible for primary care at SCF though not considered owners, and nonnative local residents in need of time-critical emergency treatment at the emergency care facility at ANMC, but not eligible for primary care at SCF. 
The proportion of these latter 2 groups is quite small; hence, we will use COs to refer to the Alaska Native and American Indian people eligible for SCF care.

After extracting monthly data for the above measures, we determined that we had insufficient events to analyze monthly rates for a subset of measures. We then ran a second round of data extraction aggregating measures on a bimonthly basis or annual basis, as necessary. We would have liked to have had more years of data before the start of the PCMH implementation, but the SCF Data Services staff characterized the inclusion of medical encounters in RPMS before 1994 as incomplete. We used a 3-year look-back to ensure that all $\mathrm{COs}$ included in the sample had previously been receiving care at $\mathrm{ANMC}$; therefore, data from 1996 were the earliest we could analyze. We analyzed data through the end of 2009, the last full year of data that was available at the time of data extraction.

We report here on trends in monthly rates for all customer-owners with at least 1 emergency care visit for any reason and for unintentional injury, and bimonthly rates for adults with asthma with at least 1 emergency care visit for asthma in the past 2 months. Adults with asthma were identified as COs aged 18 years or older with an International Classification of Diseases, Ninth Edition (ICD-9) diagnosis of 493.xx in the past 3 years, excluding COs with previous ICD-9 diagnoses indicating cystic fibrosis, lung anomalies, perinatal chronic respiratory disease, and related conditions (ICD-9 codes 277.xx, 747.21, 748.3-748.6x, 748.8, $748.9,750.3,759.3$, or 770.7$)$. Unintentional injuries were defined by ICD-9 codes 800 through $995.7 x$ excluding 960 through $979.9 x$, and 995.50 through 995.59, and excluding all admissions with ICD-9 E codes E870 through E879.9x, E930 through E959. xx, and E967 through E967.9x. Each CO was counted only once per measurement period, that is, a $\mathrm{CO}$ was included once in the numerator if they had 1 or more emergency care visits in the measurement period.

We estimated emergency care rate levels and trends using segmented regression analysis with autocorrelation. Candidate variables for the regression models included a monthly counter for the entire study period (March 1996 through December 2009) to estimate the baseline studywide trend as well as monthly counters for each of 3 discrete later study segments to estimate changes from the baseline trend: segment 1 (August 1999 through July 2000, PCMH implementation); segment 2 (August 2000 through April 2005, early post-PCMH implementation); and segment 3 (May 2005 through December 2009, later post-PCMH implementation). Each model included an intercept as well as candidate variables to estimate level changes for each of the same 3 study segments. ${ }^{9}$ Final models included candidate variables that were significant at the level of $P<.05$. Fit diagnostics including plots of residuals and white noise probabilities were evaluated for all models. To improve model fit, we included outlier indicator variables in the models for overall emergency care use and emergency care use for unintentional injuries to account for extremely low rates during 7 months in 1999 (February through August) resulting from inconsistent coding of emergency care visits and 1 month in 2001 (December). Analyses were conducted using SAS software. ${ }^{10}$

\section{Qualitative Data Collection and Analysis} Qualitative Study Participants

The goal for the qualitative data analysis was to assess how changes in health care system outcomes and service use could be explained by, or otherwise associated with, the process by which these changes have taken place in the health care system from the perspective of COs, their clinicians, and other employees, and the tribal leadership at SCF. We completed analysis of semistructured, in-depth interviews with 45 individuals for this purpose. Interviews were held with representatives of the major PCMH transition stakeholder groups: current and former $\mathrm{COs}(\mathrm{n}=11)$; primary care clinicians $(\mathrm{n}=8)_{i}$, health system employees, including administrators, support staff, and non-primary care clinicians $(\mathrm{n}=15)$; and SCF tribal leaders $(\mathrm{n}=11)$ (Table 1$)$.

Employee inclusion criteria consisted of (1) SCF employment during the primary period of the transition to a PCMH (1995-2004) and (2) holding an SCF position associated with the PCMH as determined by the SCF research team (eg, primary care clinicians, specialty care clinicians, supportive staff, administrators). To protect employee privacy, the SCF Human Resources department provided the ICHS team with a deidentified list of eligible past and present employees; each was assigned a nondescriptive, unique identification number. ICHS team members randomly selected participants' identification numbers from the sampling
Table 1. Research Teams' Responsibilities According to Stakeholder Group

\begin{tabular}{lccc}
\hline & \multicolumn{3}{c}{ Responsibility } \\
\cline { 2 - 4 } Stakeholder & Recruitment & Data Collection & Data Analysis \\
\hline Customer-owners & SCF and ICHS & SCF & SCF and ICHS \\
Tribal leaders & SCF & SCF & SCF and ICHS \\
Employees & SCF (HR) and ICHS & ICHS & SCF and ICHS \\
\hline HR = human resources; ICHS = Institute for Circumpolar Health Studies; SCF = Southcentral \\
Foundation.
\end{tabular}




\section{Table 2. Duration of Study Participants' Experience With the Nuka System of Care}

\begin{tabular}{lccc}
\hline & \multicolumn{3}{c}{ Affiliation, Years } \\
\cline { 2 - 4 } Stakeholder & $\begin{array}{c}\text { Pretransition and } \\
\text { Posttransition }\end{array}$ & $\begin{array}{c}\text { Posttransition } \\
\text { Only }\end{array}$ & Total \\
\hline Customer-owners & 10 & 1 & 11 \\
Tribal leaders & 11 & 0 & 11 \\
Employees & 6 & 17 & 23 \\
\hline $\begin{array}{l}\text { a Pretransition and posttransition, 1999. } \\
\text { b Posttransition, 1999. }\end{array}$
\end{tabular}

frame and provided the list of selected participant identification numbers to SCF human resources, which then mailed letters to randomly selected employees inviting them to participate in the study. Interested employee participants contacted the ICHS team to schedule in-depth interviews. ICHS conducted this process of random employee selection and invitations multiple times throughout the length of the study. SCF researchers recruited a convenience sample of COs using flyers posted in clinical settings and advertisements in Alaska Native and American Indian publications. SCF researchers recruited a convenience sample of tribal leaders using mailed invitations and mass e-mails. The inclusion criterion for COs and tribal leaders consisted of any interaction with the SCF health system from 1995 to 2004.

Interview participants' length of time affiliated with the Nuka System of Care varied (Table 2), providing the research team with multiple perspectives.

Although initiated in 1999, the SCF transition to a PCMH model of care was an evolving process. Growth in and change to the health care system continued thereafter and to this day (Figure 1); therefore, the stakeholders there both before and after the transition, as well as the stakeholders there only after the transition had considerable input on the continuous changes that took place throughout the study.

\section{Qualitative Data Collection}

All employee interviews were scheduled and conducted outside the ANMC/SCF facility at the University of Alaska at Anchorage to ensure employee confidentiality. COs were interviewed in private meeting rooms located at ANMC, and tribal leaders were interviewed in their private offices at SCF.

Semistructured interviews included open-ended questions about perceptions and experiences regarding the provision of care, quality of care, and access to care, as well as general satisfaction with and observations on the transformation of the health care system to a PCMH model. We used contextual probes to follow up on new or unexpected themes that emerged from the interviews. In addition to the open-ended questions described above, we used the following:

- COs: Please tell me what you remember about the health care system at SCF from 1995-1999 and 2000-2004.

- Tribal leaders: Can you tell me how the health care system has changed over the past 10 years? What was happening in the bigger picture to spur the health care changes? How did you determine the best process to implement these changes?

- Employees: Who did you consider a team member during the pretransition and posttransition time periods?

All interviews were conducted by an interviewer and note taker, 44 were in person, and 1 was conducted via telephone. The interviewer walked the participant through the informed consent form, answered any questions, elicited approval to record, and asked the key or structured questions in the guide. In addition to taking field notes and overseeing the recording process, the note taker also asked questions for clarification and followed up on missed probes at the end of the interviews. Note takers, or other members of the project team for employee and tribal leader interviews, integrated the field notes and the digital recording of the interview into summary transcripts. These transcripts were provided to the interviewer, along with a copy of the digitized recording, and the interviewer completed the review and finalized the transcript for subsequent analysis. The summary transcripts were carefully edited to ensure they did not include identifiers either of the interviewee or any employees mentioned in the interviews.

\section{Qualitative Data Analysis}

We coded and organized all interview data using NVivo 9 software (QSR International Pty Ltd). ${ }^{11}$ The analytic process consisted of 2 phases. In the first phase of coding, the unit of analysis was the participant, and the data consisted of entire interview transcripts. At least 2 separate coders reviewed and coded each interview transcript using a priori codes selected from the analytic cross-walk because of their potential to provide important contextual details related to the quantitative data. These selected codes included access to care, chronic health conditions (asthma, diabetes, and chronic obstructive pulmonary disease), communication, empanelment, emergency department use, relationships, and team-based care. We selected 2 of these codes, empanelment and team-based care, as providing the most substantive and relevant insights into quantitative trends. In addition, we augmented the coding structure hierarchically to incorporate several second- 
level codes so that additional dimensions of meaning could be examined separately for these codes.

In the second phase of coding, the unit of analysis was the a priori response code, and the data consisted of segments of summary transcripts coded as relevant to empanelment and team-based care. At least 2, and often 3, separate coders reviewed and coded each segment using the second-level codes identified in the first phase: enhanced access to care, changes in relationships and coordination of care, and leadership decision making.

The project team met to assess interrater reliability between coders and to discuss any revisions to the codebook and analytic protocol, throughout both phases of analysis. We assessed interrater reliability using the Cohen $\kappa$ and maintained $а \boldsymbol{\kappa}$ coefficient of .40 or greater between all coders throughout analysis. Although statistical analysis of interrater reliability is not always conducted for coding of qualitative data, we believed that given the composition of the coding team (university- and clinic-based researchers) and relatively small number of a priori and emergent secondary codes, a $\kappa$ statistic was appropriate for evaluating agreement between coders. ${ }^{12}$

\section{RESULTS}

\section{Quantitative Results}

The total patient study population numbered 27,831 at the beginning of the study period in March 1996 and increased to 48,043 in December 2009. This increase mirrored the increase in the Alaska Native and American Indian population in the Anchorage area during the same period. Emergency care use rate among all COs for any diagnosis (Supplemental Figure 1, avail-

able online at http://annfammed.org/content/11/

Suppl_1/S41/suppl/DC1) was increasing before the PCMH implementation $(t=3.74, P<.001)$, dropped during implementation $(t=-0.49, P<.001)$, continued to decrease steadily immediately after implementation $(t=-5.46, P<.001)$, and subsequently leveled off $(t=-3.82, P<.001)$. The total $R^{2}$ for this model was 0.90 , and the regression $R^{2}$ was 0.83 .

The population of adult asthma COs numbered 390 in March 1996 and increased to 2,823 in December 2009. Emergency care use by this group for asthma

(Supplemental Figure 2, available online at http:// Him annfammed.org/content/11/Suppl_1/S41/suppl/ DC1), as evidenced by a primary or secondary purpose of visit coded with ICD-9 codes for asthma, decreased steadily from the beginning of the study through the early post-PCMH implementation period and then flattened out during the later post-PCMH implementation period $(t=-10.36, P<.001$ for study- wide trend and $t=4.30, P<.001$ for change in trend during later post-PCMH implementation). The total $R^{2}$ for this model was 0.86 , and the regression $R^{2}$ was 0.67 .

Emergency care use among all COs for unintentional injuries (Supplemental Figure 3, available online at http://annfammed.org/content/11/Suppl_1/S41/ suppl/DC1), a control measure, showed an increasing trend before and during implementation and decreasing trends after implementation $(t=2.12, P=.04$ for studywide trend, $t=-3.38, P<.001$ for change in trend during early post-PCMH implementation, and $t=-2.92, P=.004$ for change in trend during later post-PCMH implementation). The total $R^{2}$ for this model was 0.83 , and the regression $R^{2}$ was 0.54 .

\section{Qualitative Results}

\section{Enhanced Access}

All interview participants (COs, primary care clinicians, administrators, support staff, and SCF tribal leaders) gave detailed descriptions of their experiences with the provision of enhanced access to primary care at SCF. Enhanced access refers to open (often sameday) scheduling of visits, expanded office hours, and additional options for communication between $\mathrm{COs}$ and their clinicians. Many tribal leaders and administrators detailed the resources necessary to support enhanced access to care. In the words of one tribal leader, "[Primary care] grew and we grew. We had Primary Care Center 1, which you can't hardly see any more. And it grew to Primary Care Center 1 and 2 , then 1,2 , and 3 . It grew physically in size and staff and in patronage."

Despite substantially increased resources for primary care, the majority (65\%) of primary care clinicians thought that the increased demand for primary care services during the transition to a PCMH model outpaced the rate at which resources to meet that demand were likewise increasing. For example, open access resulted in overbooking, or the addition of unscheduled daily clinical encounters throughout the day without limit. In the words of one physician, "I've seen providers cry, you know? Because they had 14 appointments at 4:30 and they had to get home to their family and we had this unlimited overbook and that's the expectation. For some teams it was really hard to manage. I think there was a lot of stress around that in the clinic." Several clinicians mentioned colleagues who were unwilling or unable to meet the demand and resigned or retired during PCMH implementation. As one nurse described it, "They had a high attrition, with the providers and probably with the nurses also and...you know $60 \%$ full, they always had openings, they didn't have full employment in their primary care center; that was a problem." 
Interview responses describing the outcomes from enhanced access may provide important insights into the reductions in emergency care and hospitalization described earlier. The problem of overbooking was reduced at SCF with the addition of new staff, including additional categories of primary care clinicians and a case manager or scheduler, to each ICT. As one tribal leader described the changes to the system, "I think that maybe the real core of it, that probably brought the most resources to really make a change, was the new hospital, 'cause it provided a much improved and expanded facility and with it came a lot of new money. I think it was $\$ 36$ million of new staffing money came along with the new building... If there wasn't a new hospital built and bringing the new resources with it, I'm not sure that we could've made the kind of changes that got made because you need that starting money to really make change. It would've been really difficult without [that money]." One CO described his experience with increased resources for enhanced access in this way: "It was a new facility and they were growing. You could see changes happening... scheduling changes were starting to happen...They started hiring more and more doctors and staff." From a different clinician's perspective, "I think [emergency department visits have] gone way down. Way down, because the patients...they get in [to see their primary care clinician], they're able to get in so much easier. I think it's gotten even better since they put the case manager or the scheduler there because that's much easier, I mean infinitely easier."

\section{Changes in Relationships and Coordination of Care}

Interview results related to changes in relationships and coordination of care refer to participants' experiences and perspectives related to changes in relationships between COs and their clinicians, or among clinicians, associated team-based coordination of primary care. From the CO's perspective, the transition to a coordinated, team-based model of coordinated care began with empanelment. Exactly one-half of the COs interviewed recalled being randomly assigned to their ICT home regardless of their stated preferences, and these participants were more likely to describe the empanelment process as confusing or frustrating. Some COs, however, found the opportunity to select their primary care clinician, and through them their ICT home, empowering. Among those COs who selected their clinician, a few chose to move rapidly from one primary care clinician's panel to another. They provided a variety of reasons for doing so, including seeking reduced examination room wait times, less restrictive prescription practices, and improved personal relationships with their clinician. Over time, SCF modified the system to recognize and identify such COs in an attempt to provide services to assist them in finding an ICT with services most conducive to their needs. The majority $(67 \%)$ of COs interviewed were generally supportive of the overall coordinated care system, describing improved communication and increased feelings of safety and trust. As one $\mathrm{CO}$ described the current system, "Now it's streamlined. One call and you're directed to who is going to help you out and then they get back to you...now, there's so many people that can help you, that can care about you that you're not...you don't stay lost."

From the perspective of primary care clinicians and other employees of the system, the transition to teambased coordination of care was first associated with the addition of new structures and positions in ICTs As one employee described the process, "We quickly, probably within the first couple of years, grew to 6 or 7 behavioral health consultants, there's 20 now in primary care." The transition to a coordinated model of care was challenging for some clinicians, who were unfamiliar with the role or abilities of behavioral health clinicians or dieticians; however, most quickly sought to transfer primary care challenges to the appropriate member of the ICT, in part to reduce their own caseload. As one physician described this process, "There was a lot of skepticism as to what they were going to be able to do at the primary care clinic. But...having that behavioral health consultant as part of the team and using them...I think that is a huge...that was one of the biggest improvements I've seen."

Changes in the structure of the ICTs were also a source of some tension for nurse specialists. Before the transition to team-based care, the primary care system at SCF had developed a specialist model of care involving nurse specialists in one of several prevalent chronic health conditions, such as asthma. As one health system administrator described the transition, "So, like diabetes nurses who would work with the diabetics. And asthma nurses who would work with the chronic asthmatics, and congestive heart failure nurses who would work with congestive heart failure patients... We made a conscious decision, in like July of 1999 that the only job for nurses in primary care would be case management, and everything else would be dismantled and abolished. So, almost overnight, we eliminated all nurse manager positions, and all kind of disease specific, so we eliminated immunizations nurses and diabetes nurses and the asthma nurses, and all these kind of things." These nurses were initially concerned about the transition to ICTs, but because of open access, they could maintain their existing relationships with these COs, which may help to explain the trend in emergency care use for asthma care beginning before, and continuing after, the transition to a coordinated-care model. 


\section{Leadership Decision Making}

Study participants described observing or experiencing leadership decision making that supported the transition from emergency care to primary care. Many clinicians described a leadership who were focused on the completion of the empanelment and team-based models according to a preexisting plan, and how that plan conflicted with the experiences or expectations of some clinicians. In the words of one physician, "Some doctors who were kind of trained in a private practice mentality had a hard time with a system that valued the patient maybe more than they valued the doctor. The doctor was just one of the peer groups that assisted in taking care of that patient population." As the transition took place, feedback mechanisms such as anonymous workforce and $\mathrm{CO}$ satisfaction surveys have allowed the leadership to adopt new processes to ameliorate some of the tensions among employees while improving efficiency for COs. As one tribal leader described the process, "... as we had the opportunity to build new environments, we became much more intentional about how we built and organized those environments and it continues to change to this day."

In addition to overcoming internal tensions, several tribal leaders described the challenges associated with finding the resources necessary to build their vision. As one leader described it, "In 1998, I believe, Indian Health Service collected about $\$ 30$ million in thirdparty revenues, and today, we're collecting about $\$ 220$ million. So that's almost a 10 times change in a 15 -year period...but the fact that we were able to get significant new money from all those sources, all in that time period really made it possible to build more facilities, to hire more staff, to provide a good service to all the people that were starting to come back to us." COs and clinicians greatly appreciated the ability of the senior leadership of SCF to find and invest the resources necessary to build the system. In the words of one physician, "You come to a place like this and they were spending money... And they're giving an awful lot of money to kind of total, total care of the patient, and the total support of the patient. And with their elderly programs and stuff, you don't get that in most of the private hospitals in town. I mean these people are doing things that don't generate a lot of cash...I think they're branching out and trying to help the people get healthier."

\section{DISCUSSION}

In this study, all reported measures of emergency care use-overall use, use for a specific chronic condition (asthma), and use for unintentional injuries — showed a decreasing trend after PCMH implementation. Before implementation, the overall use and the use for unin- tentional injuries had been increasing. Conversely, the decreased emergency care use for asthma after PCMH implementation was a continuation of a decreasing preimplementation trend.

We hypothesized that emergency care use among all SCF COs for unintentional injury would be less affected by $\mathrm{PCMH}$ implementation than emergency care use for all COs for any cause; however, we found a similar decrease in both measures after the implementation. It is possible that increased access to an empanelled ICT allowed some COs to avoid emergency care for some less severe injuries that might previously resulted in an emergency care visit. It is also possible that emergency care use rates for all Alaska Native people served by SCF decreased because of systemwide factors outside the scope of this study. We may have seen less of a decrease in use for unintentional injury if we had been able to limit the analyses to more severe injuries. We tried to limit the comparison data to motor vehicle injuries, but there were insufficient cases to conduct a meaningful analysis.

Although it is not possible, looking at the time series data alone, to establish a cause and effect relationship between the PCMH implementation and trends in health care use, the mixed method design allows us to better explain them. For example, clinicians and $\mathrm{COs}$ described enhanced access to primary care services, some COs reported requiring less emergency care because of enhanced access to primary care, and tribal leaders confirmed the investment of considerable resources to support enhanced access to care. For that reason, the combined quantitative and qualitative results are consistent with decreased emergency care use resulting from a decreased need for emergency care services due to increased availability of primary care services and same-day appointments.

Further, both clinicians and COs described a nurse-specialist model of care in which asthma nurses worked closely with people with chronic asthma before the transition to a PCMH. After the transition, these specialist nurses continued to work as members of ICTs in a team-based, coordinated care environment that allowed for increased focus on proactive control and mitigation of chronic conditions such as asthma. The combined quantitative and qualitative data are consistent with a decreasing emergency care use for asthma trend before, during, and after the transition to a PCMH due to the presence of these specialist nurses working as part of coordinated ICTs.

This study had some limitations. Because demographic data were not available, it is unknown how much the study population sex and age distribution changed during the study. Demographic changes may affect rates of emergency care use. 
The rate at which IHS beneficiaries seek services outside of ANMC, and the change in that rate during the study period, are also unknown. IHS beneficiaries with no other health insurance are most likely to use emergency care services from ANMC, but beneficiaries with additional health coverage, either private or government provided, such as Medicare or Medicaid, have other choices available to them. In 2001, the Providence Alaska Medical Center in Anchorage opened a new emergency department facility. It is not known what effect the presence of this newly updated facility had on emergency care use at ANMC. Finally, as described in the Methods section, we included outlier indicator variables in the models for overall use and use for unintentional injuries to account for extremely low rates during certain periods.

In addition to the PCMH implementation there were many changes during the study period that may have affected emergency care use, either positively or negatively, over time. Examples of such changes include the transition to new clinical facilities in 1997, and parallel transfer of statewide tertiary care from IHS to SCF and the Alaska Native Tribal Health Consortium in 1999. Additional prospective confounding factors include nationwide changes in both reimbursement and medical guidelines, and continued movement of Alaska Native people to and from the Anchorage area and other areas of the state due to high fuel costs, lack of health and social services, changes in access to subsistence foods, and availability of jobs.

Finally, because this is a secondary analysis of administrative data, we were not able to ensure that all emergency care visits were accurately entered into RPMS. Extremely low rates of emergency care use were recorded in RPMS during certain periods, as previously noted. These anomalies were attributed to changes in clinic coding rules. We adjusted for these coding differences in our analysis, but there may be other coding issues that we were not able to identify.

An important next step in this field is to better understand potential causes for the observed trends in emergency care use over time. To this end, we plan to review qualitative findings for contextual information that may support or refute alternative hypotheses that are consistent with the results of this time series analysis.
To read or post commentaries in response to this article, see it online at http://www.annfammed.org/content/11/Suppl_1/S41.

Key words: patient-centered medical home; mixed methods; health disparities; Alaska native; primary care; practice-based research

Submitted June 29, 2012; submitted, revised, November 9, 2012; accepted November 19, 2012.

Funding support: This study was supported by a grant (1R18HS19154) from the Agency for Healthcare Research and Quality.

Disclaimer: The content is solely the responsibility of the authors and does not necessarily represent the official views of the Agency for Healthcare Research and Quality.

Acknowledgments: We wish to thank the patients, clinicians, staff, and tribal leadership at SCF for their contributions to this project.

\section{References}

1. Indian Health Service (IHS). IHS fact sheets: Indian health disparities [Internet]. Rockville, MD: IHS; 2011. http://www.ihs.gov/PublicAffairs/IHSBrochure/Disparities.asp. Accessed Dec 11, 2012.

2. Crabtree BF, Chase SM, Wise CG, et al. Evaluation of patient centered medical home practice transformation initiatives. Med Care. 2011:49(1):10-16.

3. Beal A, Hernandez S, Doty M. Latino access to the patient-centered medical home. J Gen Intern Med. 2009;24(Suppl 3):514-520.

4. Eby DK. Primary care at the Alaska Native Medical Center: a fully deployed "new model" of primary care. Int J Circumpolar Health. 2007;66(1 Suppl 1):4-13.

5. Davis K, Schoenbaum SC, Audet AM. A 2020 vision of patientcentered primary care. J Gen Intern Med. 2005;20(10):953-957.

6. Kilo CM, Wasson JH. Practice redesign and the patient-centered medical home: history, promises, and challenges. Health Aff (Millwood). 2010;29(5):773-778.

7. Driscoll DL, Appiah-Yeboah A, Salib P, Rupert DJ. Merging qualitative and quantitative data in mixed methods research: how to and why not. Ecolog Environ Anthropol. 2007;3(1):19-28.

8. Rittenhouse DR, Thom DH, Schmittdiel JA. Developing a policyrelevant research agenda for the patient-centered medical home: a focus on outcomes. J Gen Intern Med. 2010;25(6):593-600.

9. Wagner AK, Soumerai SB, Zhang F, Ross-Degnan D. Segmented regression analysis of interrupted time series studies in medication use research. J Clin Pharm Ther. 2002;27(4):299-309.

10. SAS software, version 9.2 of the SAS System for Windows. Cary, NC: SAS Institute Inc; 2008.

11. NVivo qualitative data analysis software, version 9. Burlington, MA: QSR International Pty Ltd; 2010.

12. Thompson C, McCaughan D, Cullum N, Sheldon TA, Raynor P. Increasing the visibility of coding decisions in team-based qualitative research in nursing. Int J Nurs Stud. 2004;41(1):15-20. 